\title{
Advanced Numerical Simulations of Temperature Anisotropy Instabilities and Collective Interaction Processes in High-Intensity Bunched Ion Beams
}

\author{
Hong Qin, Ronald C. Davidson and Edward A. Startsev \\ Princeton Plasma Physics Laboratory, Princeton University, Princeton, NJ 08543
}

\begin{abstract}
The $\delta f$ particle-in-cell simulation method has been extended to allow the perturbation to be defined relative to any reference state, and a switching algorithm that can smoothly switch between the $\delta f$ and total- $f$ methods has been developed. The improved $\delta f$ method has been successfully applied to simulate the collective dynamics of high-intensity bunched beams. Systematic studies of the influence of finite bunch length on the spectra of collective excitations in high-intensity ion beams, and the linear and nonlinear evolution of the temperature anisotropy instability has been carried out.
\end{abstract}

Key words: high intensity beam, bunched beam, perturbative particle simulation PACS: 29.27.Bd, 41.85.Ct, 41.85.Ew

\section{Introduction}

High-intensity bunched ion beams are a key component of beam-driven inertial confinement fusion designs and beam-driven high energy density physics experiments. Even though the collective dynamics and instabilities for long coasting beams have been studied extensively,

\footnotetext{
* Supported by the U.S. Department of Energy.

${ }^{\star}$ Corresponding author: Hong Qin (hongqin@princeton.edu).
} 
our basic understanding of collective effects in high-intensity bunched beams is still very limited. In particular, collective effects induced by strong coupling between the longitudinal and transverse dynamics are of fundamental importance for the applications of high-intensity bunched beams. For the ongoing Neutralized Drift Compression Experiment (NDCX) at the U.S. Heavy Ion Fusion Sciences Virtual National Laboratory [1], it is necessary to systematically study the transverse and longitudinal coupling and its effects on collective dynamics and instabilities. The self-consistent theoretical framework for studying collective effects is provided by the nonlinear Vlasov-Maxwell equations [2-4]. A corresponding numerical method, the $\delta f$ particle-in-cell (PIC) simulation method, has been developed $[5,6]$ to solve the nonlinear Vlasov-Maxwell equations with significantly reduced noise. This theoretical and numerical framework has been successfully applied to study stable beam propagation [7], electron-ion two-stream (electron cloud) instabilities [8-14], and collective instabilities driven by large energy anisotropy [15-18] for long coasting beams.

In order to effectively simulate the collective dynamics in high-intensity bunched beams, we have developed two new improvements to the $\delta f$ PIC simulation method. First, the algorithm is extended to allow the perturbation to be defined relative to any reference state, instead of an exact equilibrium solution as required in the standard $\delta f$ simulation method $[19,9]$. This is particularly critical for high-intensity bunched beams, because exact equilibrium solution for bunched beams with temperature anisotropy does not exist [20], due the coupling between the longitudinal and transverse dynamics induced by the nonlinear space-charge force. Second, a smooth-switching algorithm is developed which can switch smoothly between the $\delta f$ and total- $f$ methods. When applying the standard $\delta f$ method to simulate high-intensity bunched beams, it is found [6] that wave-particle interactions may result in large weight growth for resonant or nearly resonant simulation particles, which in turn produces large error fields and invalidates the simulation results. The switching scheme is able to automatically switch to the total- $f$ method when the weight becomes large, and still takes full advantage of the low-noise feature of the $\delta f$ algorithm when the weight is small. With these two new improvements, we have carried out extensive numerical studies of the collective dynamics in high-intensity bunched beams. It is found that the interplay between beam intensity and finite bunch geometry introduces new collective modes, and the linear and nonlinear characteristics of the temperature anisotropy instabilities are significantly modified by the finite bunch geometry.

The paper is organized as follows. In Sec. 2, the theoretical model and the improved $\delta f$ method is described. Simulation results of collective excitations and temperature anisotropy instabilities for high-intensity bunched beams are reported in Sec. 3. 


\section{Theoretical model and the new $\delta f$ PIC algorithm}

We consider a high-intensity bunched ion beam confined in both the $r$ - and $z$ - directions by an external smooth-focusing force in the beam frame

$$
\mathbf{F}_{f o c}=-m \omega_{\perp}^{2} \mathbf{x}_{\perp}-m \omega_{z}^{2} z \mathbf{e}_{z}
$$

Here, $\omega_{\perp}$ and $\omega_{z}$ are the constant transverse and longitudinal applied focusing frequencies in the smooth-focusing approximation. In the beam frame, the dynamics of the bunched beam is described by the nonlinear Vlasov-Maxwell equations [2]

$$
\begin{gathered}
\left\{\frac{\partial}{\partial t}+\mathbf{v} \cdot \frac{\partial}{\partial \mathbf{x}}-\left[m\left(\omega_{\perp}^{2} \mathbf{x}_{\perp}+\omega_{z}^{2} z \mathbf{e}_{z}\right)+e\left(\nabla \phi-\frac{v_{z}}{c} \nabla_{\perp} A_{z}\right)\right] \cdot \frac{\partial}{\partial \mathbf{p}}\right\} f(\mathbf{x}, \mathbf{p}, t)=0 \\
\nabla^{2} \phi=-4 \pi e \int d^{3} p f(\mathbf{x}, \mathbf{p}, t) \\
\nabla^{2} A_{z}=-\frac{4 \pi}{c} e \int d^{3} p v_{z} f(\mathbf{x}, \mathbf{p}, t)
\end{gathered}
$$

where $f$ is particle distribution function in phase space, and $e$ and $m$ are the particle charge and rest mass, respectively. In the new $\delta f$ PIC algorithm for numerically solving the nonlinear Vlasov-Maxwell equations Eqs. (2)-(4), the particle's distribution is partitioned as

$$
f=\alpha f_{0}+w F
$$

where $f_{0}$ is a known reference distribution function. The coefficient $\alpha$ is a function of time and can take on values between 0 and 1 . The case of $\alpha=0$ corresponds to the total- $f$ method, and the case of $\alpha=1$ corresponds to the standard the $\delta f$ method. The perturbed distribution is constructed from the distribution function $F$ of simulation particles, the weight function $w$ in phase space, and the coefficient $\alpha$ as [6]

$$
\delta f=(\alpha-1) f_{0}+w F
$$

Because the simulation particles follow the same trajectories as the physical particles, $F$ satisfies the Vlasov equation (2) as well. But $F$ need not be the same as $f$.

From Eq. (5), it is simple to derive the governing equation for the evolution of $w$,

$$
\frac{d w}{d t}=-(g-w) \frac{1}{f_{0}}\left[\left(\frac{d f_{0}}{d t}\right)_{\delta}+\left(\frac{d f_{0}}{d t}\right)_{0}\right]+\frac{w-g}{\alpha} \frac{d \alpha}{d t},
$$


where

$$
\begin{aligned}
& \left(\frac{d f_{0}}{d t}\right)_{\delta}=-e\left(\nabla \delta \phi-\frac{v_{z}}{c} \nabla_{\perp} \delta A_{z}\right) \cdot \frac{\partial f_{0}}{\partial \mathbf{p}} \\
& \left(\frac{d f_{0}}{d t}\right)_{0}=\left\{\frac{\partial}{\partial t}+\mathbf{v} \cdot \frac{\partial}{\partial \mathbf{x}}-\left[m\left(\omega_{\beta}^{2} \mathbf{x}_{\perp}+\omega_{z}^{2} z \mathbf{e}_{z}\right)+e\left(\nabla \phi_{0}-\frac{v_{z}}{c} \nabla_{\perp} A_{z 0}\right)\right] \cdot \frac{\partial}{\partial \mathbf{p}}\right\} f_{0} .
\end{aligned}
$$

Here, $\delta \phi \equiv \phi-\phi_{0}$ and $\delta A_{z} \equiv A_{z}-A_{z 0}$ are the field perturbations relative to the reference potentials $\left(\phi_{0}, A_{z 0}\right)$, which are chosen to satisfy

$$
\begin{aligned}
\nabla^{2} \phi_{0} & =-4 \pi e \int d^{3} p f_{0}(\mathbf{x}, \mathbf{p}, t) \\
\nabla^{2} A_{z 0} & =-\frac{4 \pi}{c} e \int d^{3} p v_{z} f_{0}(\mathbf{x}, \mathbf{p}, t) .
\end{aligned}
$$

For the perturbed fields, Maxwell's equations are given by

$$
\begin{aligned}
\nabla^{2} \delta \phi & =-4 \pi e \int d^{3} p \delta f(\mathbf{x}, \mathbf{p}, t) \\
\nabla^{2} \delta A_{z} & =-\frac{4 \pi}{c} e \int d^{3} p v_{z} \delta f(\mathbf{x}, \mathbf{p}, t)
\end{aligned}
$$

where $\delta f$ is calculated from Eq. (6).

If possible, it is desirable to choose the reference state $\left(\phi_{0}, A_{z 0}, f_{0}\right)$ as an exact solution, either time-dependent or time-independent, to the Vlasov-Maxwell equations (2)-(4), such that the $\left(d f_{0} / d t\right)_{0}$ term in Eq. (7) vanishes. For most applications using the standard $\delta f$ method, $\left(\phi_{0}\right.$, $\left.A_{z 0}, f_{0}\right)$ are chosen to correspond to an equilibrium solution with $\partial / \partial t=0$. For bunched beams, if the energy is isotropic in the beam frame, the reference state can be chosen to be an exact equilibrium solution. However, for bunched beams with energy anisotropy, exact equilibrium solution does not exist due to the lack of independent longitudinal and transverse invariants of the particle dynamics [20]. In this case, we can choose a time-independent reference distribution $\left(\phi_{0}, A_{z 0}, f_{0}\right)$ that is close to a quasi-equilibrium state.

The term $g$ in Eq. (7) is defined as $g \equiv f / F$, which is a constant of the motion for each simulation particle, i.e., $d g / d t=0$, because $d f / d t=0$ and $d F / d t=0$. Therefore, $g$ is determined from the initial conditions of the simulation particles. If $F$ is initially loaded in to be the same as $f$, then $g \equiv 1$ and the distributions of physical particles and simulation particles are the same.

Different from the standard $\delta f$ method, the dynamics of $w$ is now coupled to that of $\alpha$, which can be either prescribed or determined from some rules coupled back to the amplitude of 
$w$. When $\alpha$ varies smoothly from 1 to 0 during the simulation, the $\delta f$ method is smoothly switched to the total- $f$ method. The purpose of the switch is to overcome the noise brought on by the large weight of nearly-resonant simulation particles [6]. Before the switch, the simulation still makes effective use of the low-noise feature of the $\delta f$ method for small weight to follow the detailed evolution of the unstable mode structures. When the weight function becomes large during the nonlinear phase, the low-noise advantage of the $\delta f$ method is reduced and the simulation is switched to the total- $f$ method to avoid the large noise induced by nearly-resonant simulation particles. The coefficient $\alpha$ is generally allowed to depend on phase space coordinates, such that different simulation particles will be switched at different time.

In the present study, $\alpha$ is chosen to depend only on time $t$ to realize a smooth switch for all simulation particles simultaneously. There are many ways to select the function $\alpha(t)$ to achieve the desired switching from the $\delta f$ method to the total- $f$ method; however, the simulation results should be independent of how the switch function is selected under the condition that the noise due to large weight is suppressed, because system of equations is always equivalent to the original Vlasov-Maxwell equations. For example, we can chose the switch function to satisfy

$$
\frac{d \ln \alpha}{d t}=\left\{\begin{array}{cc}
0, & t-t_{0}<0, \\
-a\left(\frac{t-t_{0}}{\tau}\right)^{n}, & 0 \leq t-t_{0}<\tau, \\
-a, & \tau<t-t_{0},
\end{array}\right.
$$

where $t_{0}$ is the starting time of the switch, and $\tau$ is the duration of the switch. The starting time $t_{0}$ can be either prescribed before the simulation is started, or the switching can be triggered automatically when the weight growth reaches a certain threshold. The power index $n$ and amplitude parameter $a$ in Eq. (14) are chosen to satisfy $a \tau /(n+1) \gg 1$, which ensures that $\alpha \simeq 0$ after the switching $\left(t-t_{0}>\tau\right)$. An alternative switching scheme, which uses the weight equation of the standard $\delta f$ method, can be found in Ref. [21].

\section{Collective excitations and instabilities for high-intensity bunched beams}

For a single-species beam, we can neglect $A_{z}$ in the beam frame because $\left|A_{z}\right| \ll|\phi|$.To investigate collective excitations in high-intensity bunched beams, we first assume that the beam distribution is isotropic in the beam. Under this assumption, thermal equilibrium is one example of an exact equilibrium solution of the Vlasov-Maxwell equations $\left(\phi_{0}, f_{0}\right)$ that 
can be used as the reference state for the $\delta f$ method. Thermal equilibrium is specified by

$$
\begin{aligned}
& f_{0}=f_{0}(H)=\frac{\hat{n}}{(2 \pi m T)^{3 / 2}} \exp \left(\frac{-H}{T}\right), \\
& H=\frac{\mathbf{p}^{2}}{2 m}+e \phi_{0}+\frac{1}{2} m\left(\omega_{\perp}^{2} r^{2}+\omega_{z}^{2} z^{2}\right) .
\end{aligned}
$$

Here, $H$ is the invariant energy, $T=$ const. is the (isotropic) temperature, and $\hat{n}$ is the beam number density at $(r, z)=(0,0)$. The equilibrium potential $\phi_{0}$ is determined self-consistently from the Poisson equation

$$
\nabla^{2} \phi_{0}=-4 \pi e \hat{n} \exp \left[-\frac{m\left(\omega_{\perp}^{2} r^{2}+\omega_{z}^{2} z^{2}\right)}{2 T}-\frac{e \phi_{0}}{T}\right],
$$

which is readily solved numerically in the assumed model geometry of a perfectly conducting cylindrical pipe with wall radius $r_{w}$. The finite bunch-length changes the characteristics of the linear eigenmodes in beams [22]. Shown in Fig. 1 are the spectra of the axisymmetric $(\partial / \partial \theta=$ 0 ) linear eigenmodes obtained from the simulations for bunched beams with normalized space-charge intensity $s_{b} \equiv 4 \pi \hat{n} e^{2} / 2 m \omega_{\perp}^{2}=0.27$, but for different bunch aspect ratios, $z_{b} / r_{b}=0.71,2.5,10$, and 100. Here $r_{b}$ and $z_{b}$ are the rms radius and half-length of the beam. The spectra are obtained by taking the fast Fourier transform (FFT) of the time history of perturbed potential at $\left(r / r_{w}, z / z_{\max }\right)=(0.15,0.25)$. Figure 1 (a) is the case where $\omega_{\perp}=\omega_{z}$, corresponding to a nearly spherical charge bunch. The spectrum in Fig. 1(a) peaks around $\omega / \omega_{\perp}=2,4,6,8 \ldots$, which is qualitatively similar to the case of an infinitely-long coasting beam [2]. This is because even though the finite bunch length introduces a new characteristic frequency in the longitudinal direction, the degenerate frequency $\omega=\omega_{\perp}=\omega_{z}$ is the only dominant characteristic frequency in the system. Because the space-charge forces depress the betatron frequency of the charged particles, the spectra peak below, instead of exactly on, the even integers. As the beam aspect ratio increases, additional eigenmodes emerge in between $\omega / \omega_{\perp}=2,4,6,8 \ldots$. For example, there are two major peaks appearing in the interval $0<\omega / \omega_{\perp}<2$ for $z_{b} / r_{b}=2.5$ [Fig. 1(b)]. These additional modes are the result of a coupling between the transverse and longitudinal dynamics induced by the finite length of the charge bunch. As the bunch length increases, more eigenmodes appear in the intervals between even integers [Fig. 1(c)]. However, as the bunch length becomes large, these additional eigenmodes congregate towards even integers [Fig. 1(c)]. When the bunch length approaches infinity, the spectrum of an infinitely-long coasting beam [2] is recovered [Fig. 1(d)]. Even though the spectra for a nearly spherical charge bunch and an infinitely-long coasting beam are qualitatively similar, we note that the similarity is merely a consequence of the degeneracy in the characteristic frequencies for the case in Fig. 1(a). The effects of finite bunch length are evident in Fig. 1 from the fact that the spectra undergo interesting 

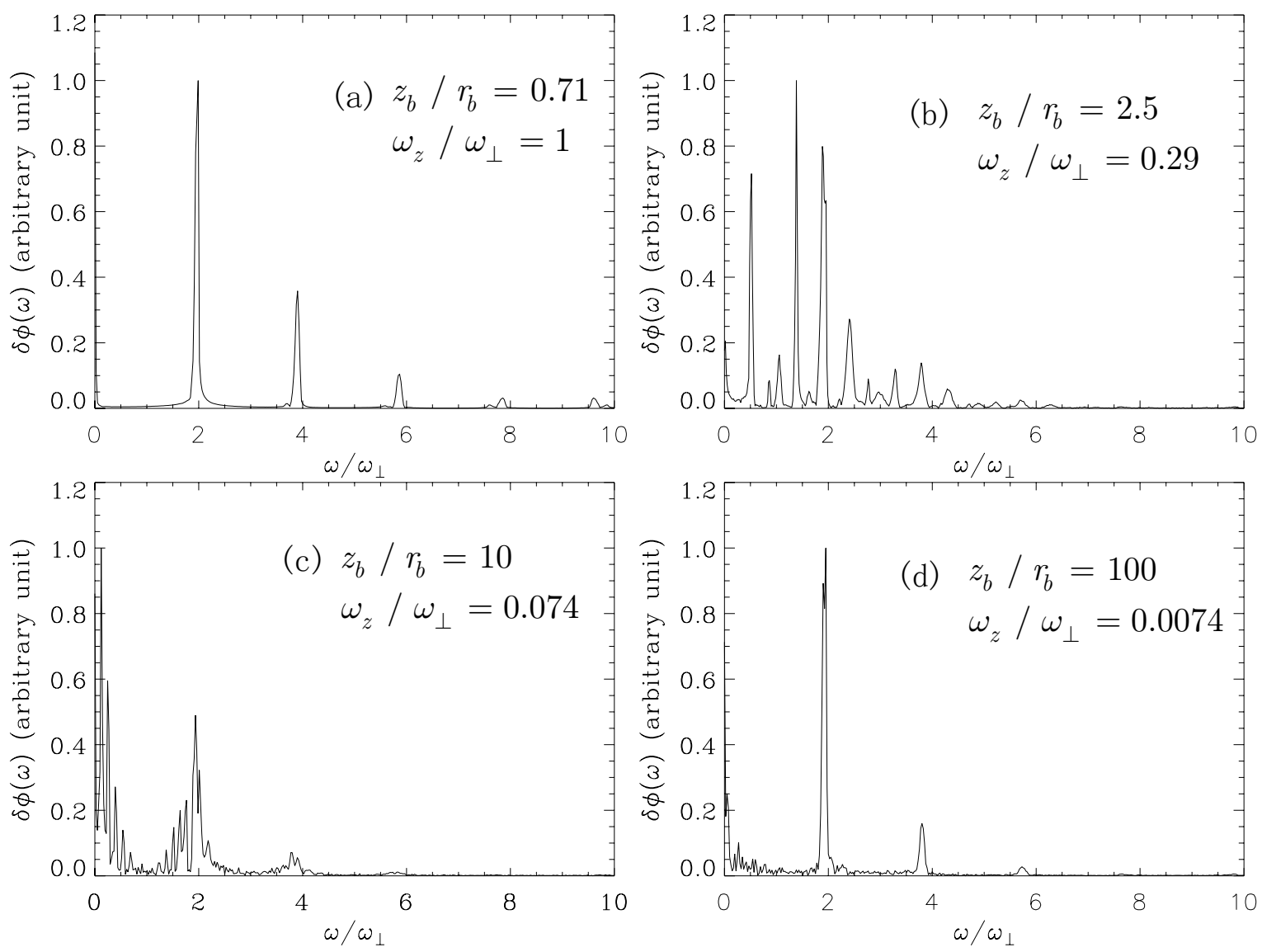

Fig. 1. Spectra of axisymmetric linear eigenmodes for bunched beams with normalized space-charge intensity $s_{b}=0.27$ and different bunch aspect ratios $z_{b} / r_{b}=0.71,2.5,10$, and 100 . The values of $r_{b} / r_{w}$ are maintained at $r_{b} / r_{w}=0.35$ for all cases. The spectra are obtained by taking the fast Fourier transform (FFT) of the time history of perturbed potential at $\left(r / r_{w}, z / z_{\max }\right)=(0.15,0.25)$.

changes when the bunch length varies between these two limiting cases.

The large energy anisotropy characteristic of charged particle beams in particle accelerators has long been thought as a possible free energy source to drive the electrostatic Harris instability [15-18]. To simulate this instability, it is desirable to start from quasi-steady equilibria with anisotropic distribution function in the transverse and longitudinal directions. However, as discussed previously [20], exact kinetic equilibria do not exist for anisotropic bunched beams. It is necessary to construct a reference state which is an approximate kinetic equilibria with anisotropic distribution function. For those cases where the transverse-longtitudinal 
coupling is weak, the transverse energy $H_{\perp}$ and longitudinal energy $H_{z}$ defined by

$$
\begin{aligned}
H_{\perp} & =\frac{p_{\perp}^{2}}{2 m}+\frac{m}{2} \omega_{\perp}^{2} r^{2}+e \widetilde{\phi_{0}}(r, z), \\
H_{z} & =\frac{p_{z}^{2}}{2 m}+\frac{m}{2} \omega_{z}^{2} z^{2}+e\left\langle\phi_{0}\right\rangle(z),
\end{aligned}
$$

are approximately conserved [20]. Here, $\left\langle\phi_{0}\right\rangle, \widetilde{\phi_{0}}$, and $\overline{\phi_{0}}$ are defined as

$$
\begin{aligned}
\left\langle\phi_{0}\right\rangle(z) & =\overline{\phi_{0}}(z)-\overline{\phi_{0}}(0), \\
\widetilde{\phi_{0}}(r, z) & =\phi_{0}(r, z)-\left\langle\phi_{0}\right\rangle(z), \\
\overline{\phi_{0}}(z) & =\frac{\int_{0}^{r_{w}} r \phi_{0}(r, z) d r}{r_{w}^{2} / 2} .
\end{aligned}
$$

As an example, we choose the reference distribution function $f_{0}$ in the beam frame to be the anisotropic thermal equilibrium distribution

$$
f_{0}=\frac{\hat{n}}{\left(2 \pi m T_{\perp}\right)\left(2 \pi m T_{z}\right)^{1 / 2}} \exp \left(-\frac{H_{\perp}}{T_{\perp}}-\frac{H_{z}}{T_{z}}\right) .
$$

Here, $T_{\perp}$ and $T_{z}$ are the constant transverse and longitudinal temperatures, respectively. The reference density profile $n_{0}(r, z)$ and reference potential $\phi_{0}(r, z)$ are determined selfconsistently from Eq. (10).

There are two terms that determine the dynamics of $w$ in Eq. (7). The $\left(d f_{0} / d t\right)_{\delta}$ term is driven by the perturbed fields, and the second term $\left(d f_{0} / d t\right)_{0}$ is the deviation of the reference state $f_{0}$ relative to an exact equilibrium solution of the Vlasov-Maxwell equations. Some straightforward algebra gives [20]

$$
\frac{1}{f_{0}}\left(\frac{d f_{0}}{d t}\right)_{0}=-\frac{\dot{H}_{\perp}}{T_{\perp}}-\frac{\dot{H}_{z}}{T_{z}}=\dot{H}_{z}\left(\frac{1}{T_{\perp}}-\frac{1}{T_{z}}\right),
$$

where

$$
\dot{H}_{z}=e v_{z} \frac{\partial \widetilde{\phi_{0}}(r, z)}{\partial z}
$$

and super-dot ( ) denotes $(d / d t)_{0}$ defined in Eq. (9). For a well-chosen reference state $\left(f_{0}, \phi_{0}\right)$, the dynamics associated with $\left(d f_{0} / d t\right)_{0}$ has a longer time-scale for variation than that of $\left(d f_{0} / d t\right)_{\delta}$.

Shown in Fig. 2(a) is the time history of an unstable, azimuthally-symmetric perturbation relative to the reference state $\left(f_{0}, \phi_{0}\right)$ at the spatial location $\left(r / r_{w}, z / z_{\max }\right)=(0.1,0.25)$ for 

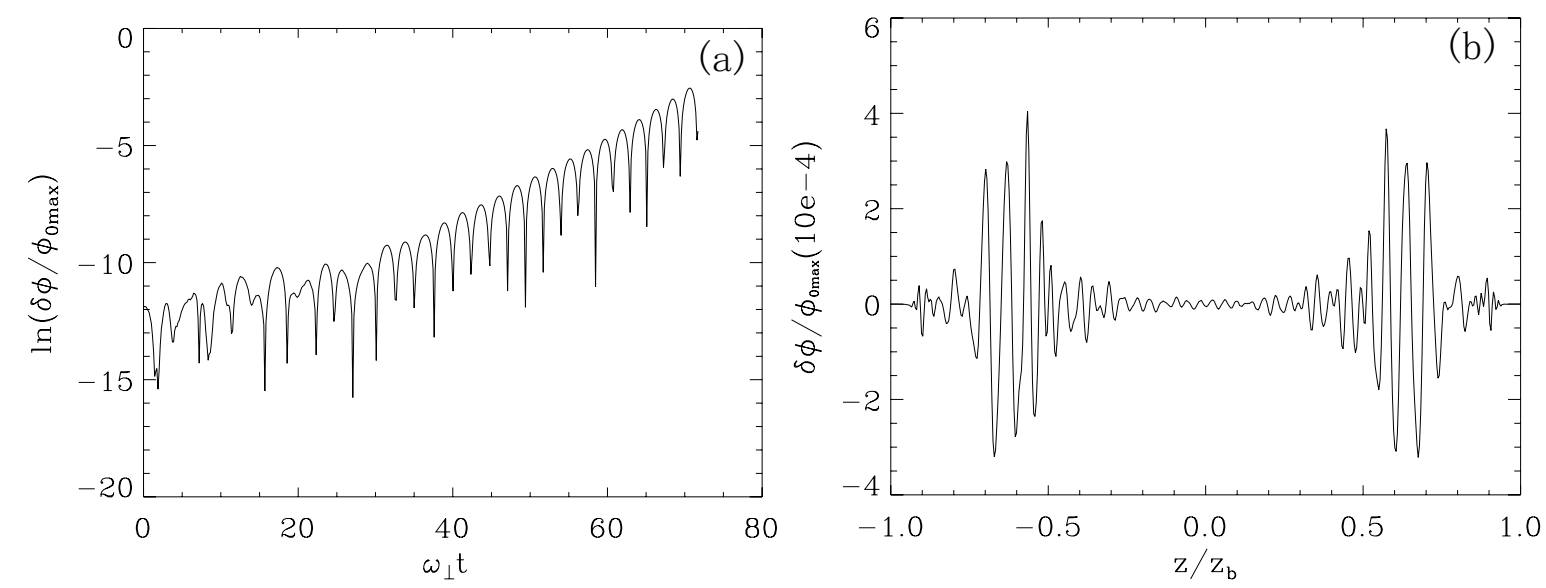

Fig. 2. (a) Time history of an unstable perturbation at one spatial location $\left(r / r_{w}, z / z_{\max }\right)=(0.1,0.25)$ for a high-intensity anisotropic charge bunch with $s_{b}=0.8$, $T_{z} / T_{\perp}=1 / 36, z_{b} / r_{b}=40$. (b) Unstable perturbation potential $\delta \phi$ plotted as a function of $z / z_{b}$ at time $t=43 / \omega_{\perp} \cdot(b)$.

a high-intensity anisotropic charge bunch with $s_{b}=0.8, T_{z} / T_{\perp}=1 / 36$, and $z_{b} / r_{b}=40$. Here $r_{w}$ is the wall radius and $z_{\max }$ is half-length of the simulation domain. The instability growth rate is measured to be $\operatorname{Im} \omega=\gamma=0.1 \omega_{\perp}$, and the real frequency is $\omega_{r}=\operatorname{Re} \omega \approx \omega_{\perp}$. The simulation presented in Fig. 2 is carried out for the linear phase of the instability, using the $\delta f$ method in the linearization approximation. Because the dynamics of the reference state associated with $\left(d f_{0} / d t\right)_{0}$ is slow in comparison with the instability evolution, the $\left(d f_{0} / d t\right)_{0}$ term is neglected to emphasize the structure of the instability during the linear stage. Note that the unstable structure localizes symmetrically in the vicinity of $z / z_{\max }=$ \pm 0.6 [Fig. 2(b)]. The localization is found to be more prominent for larger bunch length. As $z_{b} / r_{b} \rightarrow \infty$, the unstable structure becomes highly localized such that the beam intensity is approximately uniform across the unstable structure in the longitudinal direction, and the instability characteristics are asymptotic to those of long coasting beams with uniform density. This is consistent with the fact that the growth rate decreases for increasing bunch length, which has been numerically confirmed [22] .

We now turn to the long-term nonlinear evolution of the instability with dynamic background evolving from the initial reference state. Shown in Fig. 3 is the time history of the potential perturbation at $\left(r / r_{w}, z / z_{\max }\right)=(0.12,0.20)$. It is clear that the dynamics contain two time-scales. The fast time-scale dynamics corresponds to the evolution of the temperature anisotropy instability, and the slow time-scale dynamics corresponds to the background dynamics of the anisotropic reference state. The fast time-scale instability has three phases. Before $t=170 / \omega_{\perp}$ is the linear growth phase. Plotted in Fig. 4(a) is mode structure as a 


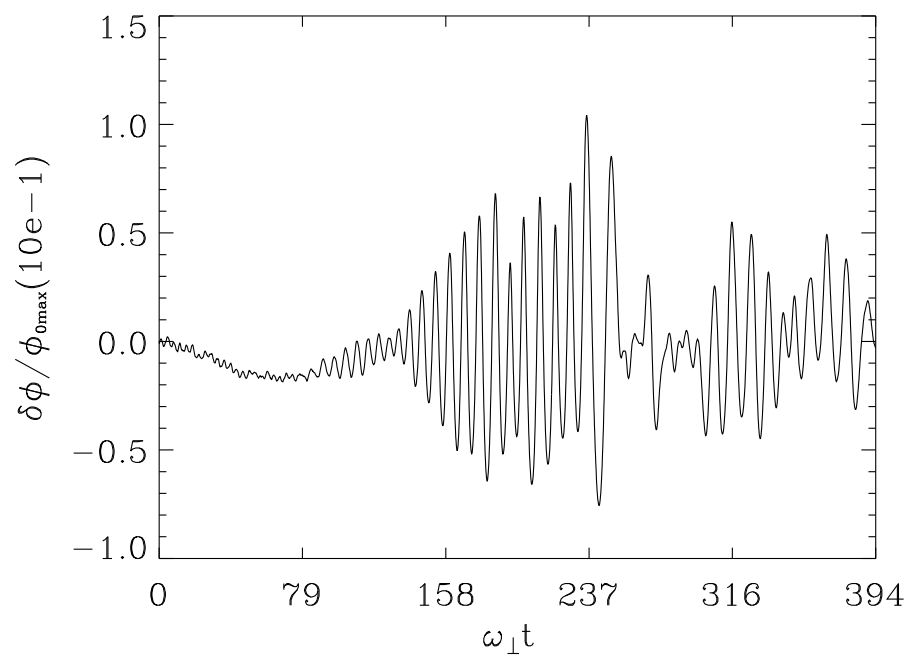

Fig. 3. Time history of an unstable perturbation at one spatial location $\left(r / r_{w}, z / z_{\max }\right)=(0.12,0.20)$ for a high-intensity anisotropic charge bunch with $s_{b}=0.8, T_{z} / T_{\perp}=1 / 36$, $z_{b} / r_{b}=40, r_{b} / r_{w}=0.26$, and $z_{b} / z_{\max }=0.40$.
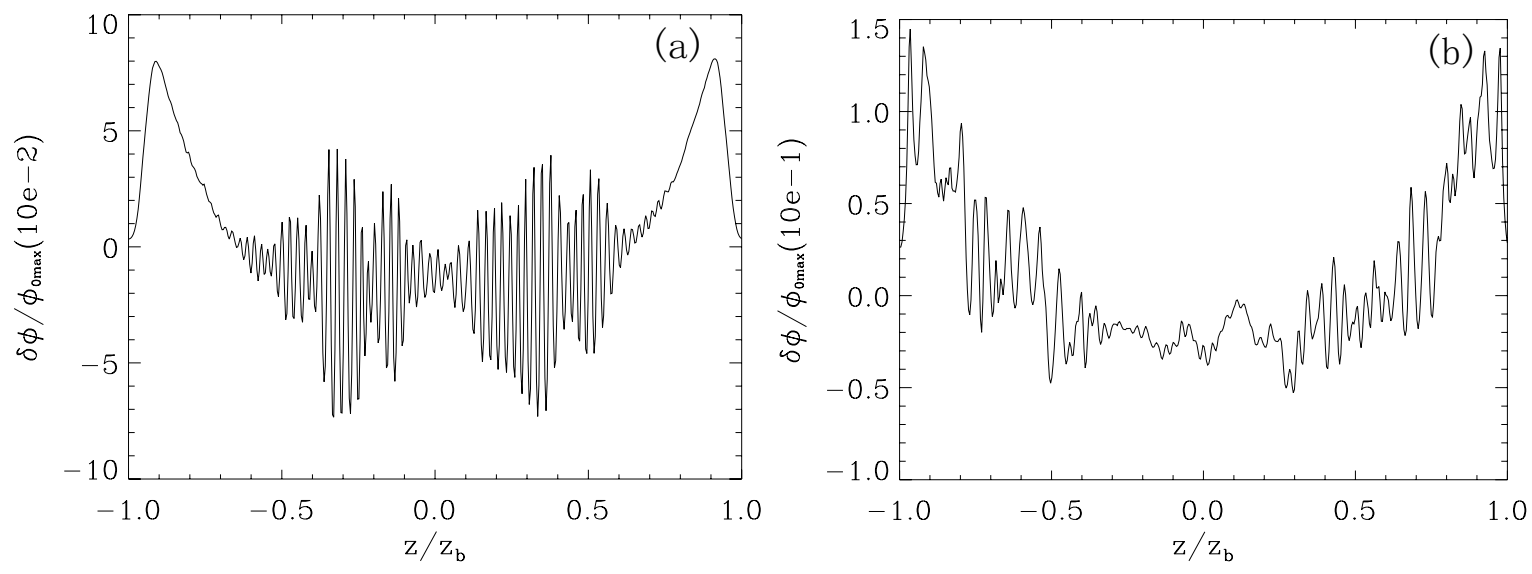

Fig. 4. (a) Unstable mode structure as a function of $z / z_{b}$ at time $t=115 / \omega_{\perp}$, which represents the linear mode structure. (b) Perturbed potential as a function of $z / z_{b}$ of the quasi-steady state at $t=390 / \omega_{\perp}$.

function of $z / z_{b}$ at time $t=115 / \omega_{\perp}$, which represents the linear mode structure. The mode structure relative to the slow time-scale background dynamics shows the same localization feature as in Fig. 3(b). The nonlinear saturation phase spans from $t=170 / \omega_{\perp}$ to $t=250 / \omega_{\perp}$, during which the amplitude of the instability nonlinearly saturates, but the perturbation maintain approximately the same frequency and spatial structure. After $t=250 / \omega_{\perp}$, the instability energy cascades partially to long wavelengths, and merges with the background 
dynamics, which eventually evolves into a quasi-steady state with the structure displayed in Fig. 4(b). The deviation of the quasi-steady state relative to the initial reference state is at the $15 \%$ level.

\section{Conclusions}

To effectively simulate the collective dynamics in high-intensity bunched ion beams, we have extended the nonlinear $\delta f$ PIC simulation method to allow the perturbation to be defined relative to any reference state, and have developed a switching algorithm which can smoothly switch between the $\delta f$ and total- $f$ methods. Using the extended $\delta f$ method, we have systematically studied the influence of finite bunch length on the spectra of collective excitations in high-intensity beams, and the linear and nonlinear evolution of the energy anisotropy instability. It is found that the finite bunch length introduces new collective modes by coupling the longitudinal and transverse dynamics through the nonlinear spacecharge force, and the spectra undergo interesting changes when the bunch-length varies. For the energy anisotropy instability, the unstable mode is symmetrically localized in two regions away from the bunch center in the longitudinal direction. Nonlinearly, the instability saturates at the $10 \%$ level, and couples to the background dynamics of the reference state. A quasi-steady state is reached at the final stage, and the deviation of the quasi-steady state relative the initial reference state is at the $15 \%$ level.

\section{References}

[1] B. G. Logan, J. J. Barnard, F. Bieniosek, C. M. Celata, R. C. Davidson, A. Friedman, E. Gilson, I. Kaganovich, J. W. Kwan, M. Leitner, A. Molvik, H. Qin, P. Roy, A. Sefkow, P. Seidl, E. Startsev, S. Yu, W. Waldron, R. Briggs, R. A. Kishek, D. R. Welch, C. Olson, Nuclear Instruments and Methods in Physics Research A 577 (2007) 1.

[2] R. C. Davidson, H. Qin, Physics of Intense Charged Particle Beams in High Energy Accelerators, World Scientific, Singapore, 2001.

[3] R. C. Davidson, I. Kaganovich, H. Qin, E. A. Startsev, D. R. Welch, D. V. Rose, H. S. Uhm, Physical Review Special Topics - Accelerators and Beams 5 (2004) 114801.

[4] R. C. Davidson, H. Qin, S. I. Tzenov, E. A. Startsev, Physical Review Special Topics Accelerators and Beams 5 (2002) 084402.

[5] H. Qin, Physics of Plasmas 10 (5) (2003) 2078-2086. 
[6] H. Qin, R. C. Davidson, E. A. Startsev, Physics of Plasmas 15 (2008) 063101.

[7] R. C. Davidson, Physical Review Letters 81 (1998) 991.

[8] H. Qin, E. A. Startsev, R. C. Davidson, Physical Review Special Topics - Accelerators and Beams 6 (1) (2003) 014401.

[9] H. Qin, R. C. Davidson, W. W. Lee, Physical Review Special Topics - Accelerators and Beams 3 (2000) 084401.

[10] R. C. Davidson, H. Qin, P. H. Stoltz, T. S. Wang, Physical Review Special Topics - Accelerators and Beams 2 (1999) 054401.

[11] R. C. Davidson, H. Qin, T. S. Wang, Physics Letters A 252 (1999) 213.

[12] R. C. Davidson, H. Qin, Physics Letter A 270 (2000) 177.

[13] H. Qin, R. C. Davidson, W. W. Lee, R. Kolesnikov, Nuclear Instruments and Methods in Physics Research Section A 464 (1-3) (2001) 477-483.

[14] H. Qin, R. C. Davidson, W. W. Lee, Physics Letters A 272 (5-6) (2000) 389-394.

[15] E. A. Startsev, R. C. Davidson, H. Qin, Physics of Plasmas 9 (2002) 3138.

[16] E. A. Startsev, R. C. Davidson, H. Qin, Physical Review Special Topics - Accelerators and Beams 6 (2003) 084401.

[17] E. A. Startsev, R. C. Davidson, H. Qin, Physical Review Special Topics - Accelerators and Beams 8 (2005) 124201.

[18] E. A. Startsev, R. C. Davidson, H. Qin, Physics of Plasmas 14 (2007) 056705.

[19] S. E. Parker, W. W. Lee, Physics of Fluids B 5 (1993) 77.

[20] H. Qin, R. C. Davidson, E. Startsev, Nuclear Instruments and Methods in Physics Research A $572(2007) 86$.

[21] E. Belova, R. Davidson, H. Ji, M. Yamada, Physics of Plasmas 11 (2004) 2523.

[22] H. Qin, R. C. Davidson, E. A. Startsev, Physical Review Special Topics - Accelerators and Beams 10 (2007) 064201. 